\title{
A Bayesian data assimilation framework for lake 3D hydrodynamic models with a physics-preserving particle filtering method using SPUX-MITgCm v1
}

\author{
Artur Safin ${ }^{1}$, Damien Bouffard ${ }^{1}$, Firat Ozdemir ${ }^{2}$, Cintia L Ramón ${ }^{3,1}$, James Runnalls ${ }^{1}$, Fotis Georgatos ${ }^{2}$, \\ Camille Minaudo ${ }^{4}$, and Jonas Šukys ${ }^{1}$ \\ ${ }^{1}$ Eawag: Swiss Federal Institute for Aquatic Science and Technology, Switzerland \\ ${ }^{2}$ Swiss Data Science Center, Switzerland \\ ${ }^{3}$ Water Research Institute and Department of Civil Engineering, University of Granada, Spain \\ ${ }^{4}$ École Polytechnique Fédérale de Lausanne, Switzerland
}

Correspondence: Artur Safin (artur.safin@eawag.ch)

\begin{abstract}
We present a Bayesian inference for a three-dimensional hydrodynamic model of Lake Geneva with stochastic weather forcing and high-frequency observational datasets. This is achieved by coupling a Bayesian inference package, SPUX, with a hydrodynamics package, MITgcm, into a single framework, SPUX-MITgcm. To mitigate uncertainty in the atmospheric forcing, we use a smoothed particle Markov chain Monte Carlo method, where the intermediate model state posteriors

5 are resampled in accordance with their respective observational likelihoods. To improve the assimilation of remotely sensed temperature, we develop a bi-directional Long Short-Term Memory (Bi-LSTM) neural network to estimate lake skin temperature from a history of hydrodynamic bulk temperature predictions and atmospheric data. This study analyzes the benefit and costs of such state of the art computationally expensive calibration and assimilation method for lakes.
\end{abstract}

\section{Introduction}

Lake management is a constantly evolving trade-off between different conflict of use. The most obvious is that lakes are easily accessible sources of drinking water but also the place where wastewater is ultimately discharged. Lake stakeholders traditionally evaluate the evolution of lakes from in situ observations. While still not widely used for this purpose, previous studies clearly showed the benefit of one and three dimensional hydrodynamic models to project different scenarios for the short or long-term future (Gaudard et al., 2019; Soulignac et al., 2019; Vinnå et al., 2021).

While a number of dedicated monitoring projects already exist for a number of large lakes, operational fully three-dimensional (3D) models are yet quite sparse. The most notable is the NOAA Great Lakes Operational Forecast System (GLOFS) (Chu et al., 2011; Anderson et al., 2018), which provide comprehensive predictions (water temperature, velocity and level, and ice cover) for all the Laurentian Great Lakes. Over 25 years, the forecasting service has been continuously improved with better and more sophisticated models. Currently, data assimilation is used for calibration only (Anderson et al., 2018), but there is research toward making it part of the operational mode as well (Ye et al., 2020). Another platform is Meteolakes, which provides short-term water temperature and velocity forecasts for Lakes Geneva, Biel, Zurich and Greifen in Switzerland (Baracchini, 
2019; Baracchini et al., 2020a, b). The platform uses an ensemble Kalman filter to assimilate remotely sensed lake surface water temperature (LSWT), which reduced the mean temperature prediction error by half. An additional benefit of the ensemble filter was a better prediction of mesoscale physical processes such as gyres and upwellings (Baracchini et al., 2020a). However, due to the limitation of the assimilation scheme, only a fraction $(\approx 3.7 \%)$ of the available LSWT images were used.

The purpose of this study is to investigate a novel approach to data assimilation of highly heterogeneous data using Bayesian inference techniques applied to a 3D hydrodynamic model of Lake Geneva. The model relies on the ensemble affine invariant sampler (EMCEE) (Goodman and Weare, 2010; Šukys and Bacci, 2021) to calibrate distributions of physical model parameters. The advantage of this approach over standard inference methods is that it provides a more informative and accurate parameter estimation, albeit at higher computational expense. To increase the confidence in the sampling algorithm, we used a particle method that provides trajectories consistent with the hydrodynamic model (Andrieu et al., 2010; Šukys and Bacci, 2021). In particular, as a substitute to the more well-known Kalman Filter and the 4D-Var algorithms, the trajectories themselves are resampled based on their respective observational likelihoods, with the more probable realizations stochastically forming the basis for sequential predictions.

To facilitate the assimilation of remotely sensed lake surface water temperature (LSWT), we deploy a Bi-directional Long Short-Term Memory (Bi-LSTM) neural network to estimate the skin temperature of the lake and to quantify its uncertainty. The network relies on a 27-hour history of hydrodynamic model bulk temperature and atmospheric predictions as inputs for the conversion. The neural network was trained using 28 months of data (2018, 2020 and Jan-Apr 2021) using MeteoSwiss COSMO-1 atmospheric model reanalysis and Meteolakes water bulk temperature predictions. We obtained a 33\% root-mean square error (RMSE) reduction with more precise uncertainty estimates in comparison to the model presented in Lieberherr and Wunderle (2018).

We present the openly available SPUX-MITgcm framework, which integrates the Bayesian inference algorithms of the SPUX package (Šukys and Bacci, 2021) with the hydrodynamics of the MITgcm code (Adcroft et al., 1997) and the trained BiLSTM network. To the best of our knowledge, the data assimilation and particle filtering approach that we propose in this paper have not been previously tested for fully three-dimensional models due to the relatively high computational costs of model parameter calibration and lack of supporting software. The results of this framework demonstrate the viability of this approach and serve as a proof of concept for other higher-dimensional problems.

\section{Data and Numerical Model}

In this section, we describe the available data, the hydrodynamic model and our data assimilation approach. As data and software reproducibility are essential to more open and accessible research, in the supplementary material, we provide documentation on accessing and running the numerical model, which enables a full replication of the results over a short period of time we present in this paper. 
Table 1. Hydrodynamic model parameters.

\begin{tabular}{|l|c|c|}
\hline Parameter & Value & Units \\
\hline \hline Quadratic bottom drag coefficient $C_{D}$ & 0.0025 & none \\
\hline Constant salinity value & 0.05 & $\mathrm{psu}$ \\
\hline Coriolis parameter $f_{0}$ & $1.068 \cdot 10^{-4}$ & $1 / \mathrm{s}$ \\
\hline Background vertical viscosity & $10^{-6}$ & $\mathrm{~m}^{2} / \mathrm{s}$ \\
\hline Background vertical diffusivity & $1.4 \cdot 10^{-7}$ & $\mathrm{~m}^{2} / \mathrm{s}$ \\
\hline Adams-Bashforth & 0.03 & $\mathrm{none}$ \\
\hline Non-dimensional lateral eddy viscosity & $6 \cdot 10^{-4}$ & $\mathrm{none}$ \\
\hline Lateral eddy diffusivity & 0.5 & $\mathrm{~m}^{2} / \mathrm{s}$ \\
\hline
\end{tabular}

\subsection{Study site}

Lake Geneva is the largest freshwater lake in Western Europe located on the border between France and Switzerland covering an area of approximately $580 \mathrm{~km}^{2}$ with an average depth of $154 \mathrm{~m}$. Spanning $73 \mathrm{~km}$ along its longest axis and with a maximum width of $14 \mathrm{~km}$, the lake consists of a wider and deeper main portion in the east and a narrow and shallow portion in the west. The water level and discharge rate into the Rhône river are managed by a dam on the western end of the lake. Lake Geneva is predominantly vertically stratified in density due to temperature, although complete mixing does occur every few years. The mountainous nature of the region significantly affects the wind patterns over the lake, with north-east and south-west being the prevalent directions. These wind patterns, along with seasonal variability in light penetration depth, significantly affect the thermal structure of the lake (Bouffard and Lemmin, 2013; Bouffard et al., 2018). As the mean water residence time in the lake is 10 years, the primary factor driving the lakes' dynamics is the atmospheric forcing.

\subsection{Hydrodynamic model}

We simulate the hydrodynamics of Lake Geneva using the MITgcm (Adcroft et al., 1997) package (tag 'checkpoint67q'), which uses the finite volume method to solve the incompressible Navier-Stokes equations under the Boussinesq approximation. Alternative packages were FVCOM (Chen et al., 2006) used by GLERL, and Delft3D-FLOW (Deltares, 2013) used by Meteolakes. We use a hydrostatic formulation combined with a third-order direct space-time flux limiter advection scheme (Prather, 1986). A nonlinear equation of state by McDougall et al. (2003) is applied with constant salinity. Due to the large size of the lake, the Coriolis force is included. A detailed list of fixed model parameter values is provided in Table 1.

The simulations are performed on a Cartesian grid ( $z$-coordinate system) with $1 \mathrm{~km}$ horizontal resolution and 50 vertical layers that gradually increase in thickness from 1 meter at the surface to 21 meters in the deepest portion of the lake. We chose a timestep of 60 seconds. While larger timesteps were still numerically stable, a smaller value was helpful in reducing vertical 
temperature over-diffusion into the deep layers. To improve the accuracy of the topography and reduce spurious artifacts near the bottom, "shaved" cells are allowed. We apply free-slip boundary conditions to both horizontal and vertical boundaries and use non-dimensional bottom drag coefficient from Bouffard and Lemmin (2013) to enable energy dissipation. On the surface, we use an implicit free surface formulation.

We model the vertical mixing processes using the nonlocal K-Profile Parameterization (KPP) scheme (Large et al., 1994), which is commonly used in oceanography. Small background vertical diffusivity and viscosity parameters are included to ensure stability (see Table 1). For the equivalent parameters on the lateral scales, we manually tuned eddy viscosity and diffusivity parameters by minimizing the difference between model predictions and in situ temperature profiles at Buchillon station. While a more optimal approach is to infer these parameters, our data assimilation scheme found them difficult to identify (see supplementary material).

Surface forcing inputs were derived from the MeteoSwiss COSMO-E numeric weather prediction model which are made at $2.2 \mathrm{~km}$ resolution. While the COSMO-E model generates an ensemble of 21 predictions, in previous hydrodynamic models of Lake Geneva only the mean and spread were used (Baracchini et al., 2020b; Cimatoribus et al., 2018). In Sect. 2.4.2, we describe a data assimilation approach that makes use of the individual ensembles, which represent the span of weather dynamics more accurately. This approach has the additional advantage of not requiring the estimation of the spatio-temporal noise parameters that Baracchini et al. (2020b) used to add stochasticity to their model. Air pressure, air temperature, wind velocity, longwave radiation, relative humidity and cloud coverage are used to determine the input fields in accordance with Fink et al. (2014), where the wind-drag coefficients of Wüest and Lorke (2003) are used to improve surface stress coupling at low wind speeds. We also include the inflow and outflow of the Rhône river using the volume flow and temperature measured a few kilometers upstream at Porte du Scex (Station Federal Office for Environment, FOEN). As smaller tributaries and precipitation/evaporation are not taken into consideration in the model, the water level in the model is manually adjusted to the measured values from the St. Prex station.

Correct transfer of heat and energy from the atmosphere is an essential component of a well-performing hydrodynamic model, especially in the summer. In this regard, the bulk transfer coefficient of sensible heat (Dalton number) is a significant parameter that in several studies (Verburg and Antenucci, 2010; Baracchini, 2019; Rahaghi et al., 2018) has been shown to be larger than the default values used in ocean simulations. Therefore, we seek to infer this parameter. In addition, to more realistically accommodate fluctuations in water transparency, we estimate a spatially uniform Secchi depth using the photosynthetically active radiation (PAR) data from the LéXPLORE moorings (Wüest et al., 2021) to determine the attenuation rate of shortwave energy in the water column. In the future, given the spatial variability of Lake Geneva (Bouffard et al., 2018; Soulignac et al., 2019), a better approach might be to use remote sensing data to estimate Secchi depth for different lake locations. Finally, we use the albedo formula of Cogley (1979) to account for seasonal changes in surface reflectivity. The Secchi measurements and albedo values are visualized in the supplementary material. 
Table 2. Characteristics of the in situ datasets. Note that we assume the FOEN river and water level data to be exact.

\begin{tabular}{|c||c|c|c|c|c|}
\hline Dataset & Physical quantity & Frequency used in the model & $\begin{array}{c}\text { Depth } \\
\text { span }(\mathrm{m})\end{array}$ & $\begin{array}{c}\text { Sensor } \\
\text { count }\end{array}$ & $\begin{array}{c}\text { Sensor } \\
\text { uncertainty }\end{array}$ \\
\hline \hline Buchillon & Temperature & hourly & 1,35 & 2 & $0.1^{\circ} \mathrm{C}$ \\
\hline LéXPLORE & Temperature & hourly & $0.25-90$ & 16 & $0.1^{\circ} \mathrm{C}$ \\
\hline LéXPLORE & Velocity magnitude & hourly & $15-90$ & 8 & $0.08 \mathrm{~m} / \mathrm{s}$ \\
\hline GE3 & Temperature & $1-2$ measurements per month & $2.5-50$ & 8 & $0.1^{\circ} \mathrm{C}$ \\
\hline SHL2 & Temperature & $1-2$ measurements per month & $2.5-290$ & 16 & $0.1^{\circ} \mathrm{C}$ \\
\hline FOEN Rhone inflow/outflow & Temperature, volume flow & hourly & - & 2 & - \\
\hline FOEN St.Prex & Water Level & hourly & surface & 1 & - \\
\hline LéXPLORE & PAR & daily & $0-30 \mathrm{~m}$ & 4 & - \\
\hline
\end{tabular}

\subsection{Observational datasets}

A particular advantage of the Bayesian framework is the natural ability to handle multiple sources of data with their respective uncertainties. For Lake Geneva, the observations are either in the form of an in situ measurement or remotely sensed surface temperature.

\subsubsection{In situ}

In situ datasets used in the simulations are summarized in Table 2 and their locations are displayed in Fig. 1. To make the data assimilation process much more manageable, the data from LéXPLORE and BAFU have been subsampled to the hourly rate from the original intervals of 5 seconds and 10 minutes, respectively. The vertical resolution of the LéXPLORE dataset was also reduced to match the model discretization levels. Finally, due to the coarse horizontal resolution of the model, only the magnitude of velocity was considered as means of calibrating the kinetic energy of the lake.

\subsubsection{Remotely sensed temperature}

A processing chain from the University of Bern enables the extraction LSWT images at a resolution of $1 \mathrm{~km}$ from the orbital Advanced Very High Resolution Radiometer (AVHRR). On average, typically 10 images of Lake Geneva are generated per day, of which around 2 are deemed usable by the retrieval process. The quality of an individual snapshot is affected by a number of factors, such as the zenith angle, cloudiness or sensor errors (Riffler et al., 2015; Kilpatrick et al., 2001). In Lieberherr and Wunderle (2018), a system of assigning a quality flag (QF) for the different satellite measurement conditions was developed. An analysis based on in situ lake data provided an estimate of the uncertainties and biases, ranging from $1.3{ }^{\circ} \mathrm{C}$ for $\mathrm{QF} 6$ to $1.5{ }^{\circ} \mathrm{C}$ for $\mathrm{QF}$ 1. However, we believe that a more accurate uncertainty model can be established, and therefore in Sect. 2.4 .3 we detail an alternative approach based on machine learning that uses a history of model predictions and weather conditions to generate a bulk-to-skin estimate together with the associated uncertainty. 


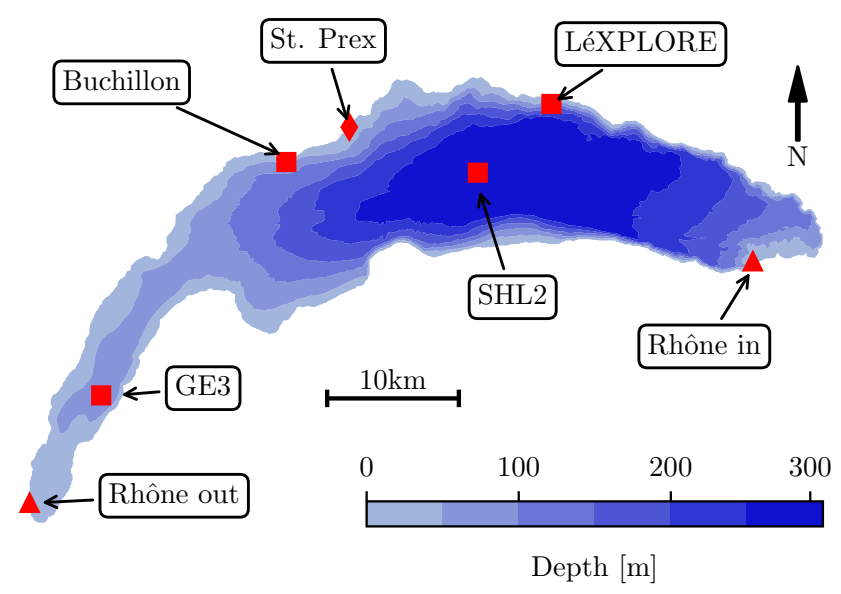

Figure 1. Location of the measurement sensors on Lake Geneva. The plot also shows the Rhone inflow and outflow sensor locations, as well as the St. Prex station, which measures the water level.

\subsection{Data assimilation}

Lakes, akin to the atmosphere, are highly volatile and sensitive systems. For hydrodynamic models, this means that small perturbations in state variables can significantly affect the resulting model trajectory. Due to the multiple sources of uncertainty present in numerical discretization models, most importantly uncertainty in forcing terms, such trajectory deviations are ultimately unavoidable. The remedy comes in the form of data assimilation (DA), a framework for providing trajectory corrections based on actual observations.

\subsubsection{Choice of DA model}

In meteorological and aquatic forecasting models, DA is an essential tool for improving predictive performance (Lahoz and Schneider, 2014; Ye et al., 2020; Baracchini et al., 2020b). A variety of DA algorithms have been proposed and deployed in operational setting, and offer different performance-to-optimality trade-offs. The two primary subfields are variational DA and sequential methods (Lahoz and Schneider, 2014). In the variational approach, an objective function describing the discrepancy between the model and observations is sought to be minimized. 4D-Var is the most popular form and offers the capability to transfer information from observed regions to unobserved for non-linear models. Some of the drawbacks of this approach are the relatively large numerical costs both for the model and uncertainty, reduced flexibility, and complex implementation of time-dependent parameters (Lahoz and Schneider, 2014; Baracchini et al., 2020b). From the sequential methods, Kalman filters $(\mathrm{KF})$, which iteratively evolve a forecast together with error covariance matrices, are an optimal approach. As the true KF is expensive due to the cost of computing the covariance matrix in model space, local model linearity is frequently assumed. For high-dimensional systems, this however can result in significant errors in the state and covariance estimation. 
Significant performance enhancement is enabled through ensemble methods, where a collection of model states are propagated and the resulting trajectories enable the estimation of covariance. Ensemble data assimilation is particularly attractive, as it significantly relaxes the requirements from the model. The ensemble KF (EnKF) is an efficient and highly popular blend, providing greater stability and easier covariance estimation. The EnKF has successfully been applied to hydrodynamic forecasting of Lake Geneva by Baracchini et al. (2020a), with a 54\% reduction in temperature error in comparison to an unassimilated model. In recent years, the local ensemble transform KF (LETKF) has been tested in a number of weather prediction frameworks with encouraging results (Gustafsson et al., 2018).

The assimilation techniques introduced above have the limitation of assuming that uncertainties and model states are Gaussian and the model is linear (Lahoz and Schneider, 2014). While this assumption is perfectly reasonable for many applications, non-Gaussian observational error and parameter distributions can be problematic for such methods. For higher resolution models, the traditional approaches of 4D-Var and EnKF have shown declining performance at convective scale (Gustafsson et al., 2018; van Leeuwen et al., 2019). To mitigate these challenges, particle filter (PF) methods have been proposed, which allow non-Gaussian distributions and are particularly suitable for non-linear models. The main challenge of PFs is to prevent filter degeneracy through an appropriate resampling technique.

In our model, we use the particle Markov Chain Monte Carlo (MCMC) method (Andrieu et al., 2010; Šukys and Bacci, 2021), which is highly suitable for non-linear problems. The MCMC algorithm is used to infer selected hydrodynamic model parameters (see Sect. 2.4.2), with ensemble affine invariant sampler (EMCEE) for the parameter acceptance/rejection criterion. For each proposed parameter set, the PF is used for data assimilation. A visualization of the process is shown in Fig. 2. To prevent filter collapse, we resample posterior trajectories according to their respective observational likelihoods before each sequential prediction. To the authors best knowledge, this is the first application of such a filtering algorithm to a fully three-dimensional model. A particular benefit of this approach is that the stochasticity from the atmosphere is sufficient to generate trajectories that manage to track the observational data with proper model parameters. This is in contrast to the non-physical correction vector in many other DA schemes that are necessary to nudge trajectories toward the data. Aside from potentially causing instabilities, these approaches decrease the confidence in the fidelity of the underlying model, as the correction mechanism potentially also corrects a model deficiency.

\subsubsection{Implementation using SPUX and design of numerical experiments}

For data assimilation and particle filtering we use the SPUX package (Šukys and Bacci, 2021), a modular framework for parallel Bayesian inference with a user-friendly programming interface. The 3D hydrodynamics package, MITgcm, was modified both to allow a Secchi depth value argument for every simulation hour and built as a shared library to enable interfacing with SPUX using the ctypes package. The ctypes approach, as opposed to launching the model as a subprocess, provided a noticeably faster and more stable performance. During calibration EMCEE was configured to run with 16 chains (distributed over 8 parallel workers), with 10 particles per filter; requiring a total of 89 parallel workers. The simulations were run on the Swiss Supercomputing Center (CSCS) over a period of approximately three months ( $\approx 10.5$ hours to predict 11 months using the $\mathrm{PF}$, and $\approx 21$ hours for a full EMCEE sampler iteration). 


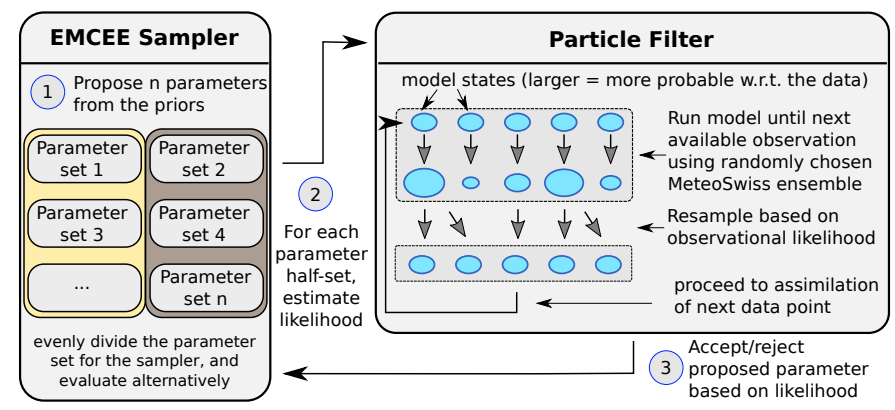

Figure 2. A visualization of the data assimilation framework. The EMCEE sampler proposes $n$ parameters, whose likelihood is then evaluated using the particle filter and used to determine whether to update that particular parameter.

As described in Sect. 2.2, we chose two hydrodynamic model parameters to infer. In both cases, we can establish an interval $(a, b)$ that contains the optimal value, but otherwise assume a uniform distribution, $U$, within. The first parameter is the Smagorinsky harmonic viscosity coefficient $C_{\text {smag }}$, to which we assign a prior distribution $U(2,4)$, consistent with Griffies and Hallberg (2000). To the second parameter - the Dalton number, $C_{\mathrm{D}}$ - we assign the prior $U(0.045,0.06)$ based on a preliminary sensitivity analysis. For Dalton values outside the prior, heat exchanges with the atmosphere were not modeled satisfactorily. For example, the default MITgcm value $C_{\mathrm{D}}=0.0346$ results in significant temperature under-prediction during the summer months.

Observational data spanning January 15 - December 15, 2019 is used for the calibration and data assimilation (DA) run. Attempts to calibrate model parameters using a shorter timeframe generated posteriors that provided sub-optimal performance for the whole year (see supplementary material for more discussion), and therefore discarded. To analyze the effectiveness of model predictions, a control run (CR) is made without filtering and using parameters $C_{\mathrm{D}}=0.045$ and $C_{\text {smag }}=2$ as the baseline.

\subsubsection{Bulk-to-Skin conversion using LSTM}

As the AVHRR operates in the infrared portion of the spectrum, it effectively measures the skin temperature in the top few millimeters of the lake. In the bulk region immediately below this surface layer, the temperature can be significantly different due to a number of factors such as wind and solar radiation (Wick et al., 1996; Alappattu et al., 2017). As the hydrodynamic model generates bulk temperature predictions, a bulk-to-skin (or skin-to-bulk) function is necessary. The available research, (e.g., Alappattu et al., 2017), generates estimates based on oceanographical studies. However, such approaches do not directly translate to lake research due to the differences in typical weather conditions, in particular, frequent low wind conditions over lakes (Bouffard and Wüest, 2019).

Thus we implement a Bi-directional Long Short-Term Memory (Bi-LSTM) neural network which uses a 27 hour history of 18 feature inputs to make a skin temperature prediction. 16 of the features come from the means and their respective spreads of the MeteoSwiss weather predictions (air temperature, cloud cover fraction, wind velocity, relative humidity, precipitation, short-wave and long-wave radiation). The last two features are the hydrodynamic model temperature predictions and hour of 
day. The model was trained using data from 2018 and January 2020 - May 2021, with the bulk water temperature predictions extracted from the Meteolakes model (Baracchini et al., 2020b).

For the particle filter, uncertainty quantification of the predicted skin temperature is also necessary. Therefore, the Bi-LSTM model also implements additional methods to quantify epistemic and aleatoric uncertainty (Kendall and Gal, 2017). Monte Carlo dropout approximates predictions from an ensemble that can be used to quantify epistemic uncertainty. On the other hand, using the negative log-likelihood of a normal distribution as the objective function in training allows Bi-LSTM to also estimate predicted variance that can be used to quantify aleatoric uncertainty. We generate 19 different skin temperature estimates and predicted variance for each input, from which we sum the average predicted variance and variance of the skin temperature estimates to obtain a scalar total variance. Accordingly, we construct a normal distribution with the computed total variance centered at the mean skin temperature prediction to be evaluated against the LSWT measurement.

\section{Results and Discussion}

In this section, we report on the inference results, with the initial focus on model parameter inference. As the calibration mechanism operates on distributions, not scalar quantities, the process is more complicated than what is typically done. Therefore this warrants a closer look at the posterior distributions and diagnostics. Then we compare the results obtained using the best posterior parameter set, and finally we evaluate the performance of the Bi-LSTM network as a predictor of skin temperature.

\subsection{Hydrodynamic model calibration}

The posterior distribution of two hydrodynamic model parameters (Smagorinsky viscosity and Dalton number) were estimated in SPUX using the EMCEE sampler (see Fig. 2). For each of the 16 parameter sets, the EMCEE sampler updates a parameter in case a better-performing one is found or it is deemed to be 'stuck' (no changes for 10 iterations). The PF is not guaranteed to choose the optimal global trajectory for a parameter set, given the computational constraints and the resetting of 'stuck' chains (Šukys and Bacci, 2021), and therefore some uncertainty around the optimal parameter value is to be expected. In Fig. 3, we show the evolution of the Markov chain parameters in terms of their means and 5\%-95\% percentiles. We can observe from the relative stationarity of the distributions that the convergence to the true posterior distribution was likely achieved. We thus conclude that the optimal model parameters were localized with a sufficiently high degree of confidence.

The inferred marginal posterior distributions are shown in Fig. 4 in orange, with the prior distribution shown in blue. The vertical red dashed lines indicate the best-found parameter set, with the values shown in the table to the right. In relation to their respective priors, the Dalton number is predicted with a relatively high degree of confidence, while the posterior for the Smagorinsky parameter is less defined. This is to be expected, as the Dalton number has a stronger effect on model predictions, and is therefore more sensitive. However, with more iterations, we expect that a more centered posterior for the Smagorinsky viscosity parameter would have been obtained.

We also consider the average redraw rate - the fraction of particles that form a basis for each sequential prediction - in Fig. 5. The survival rates dip significantly in cases when remote sensing data is assimilated or LéXPLORE data is available. 

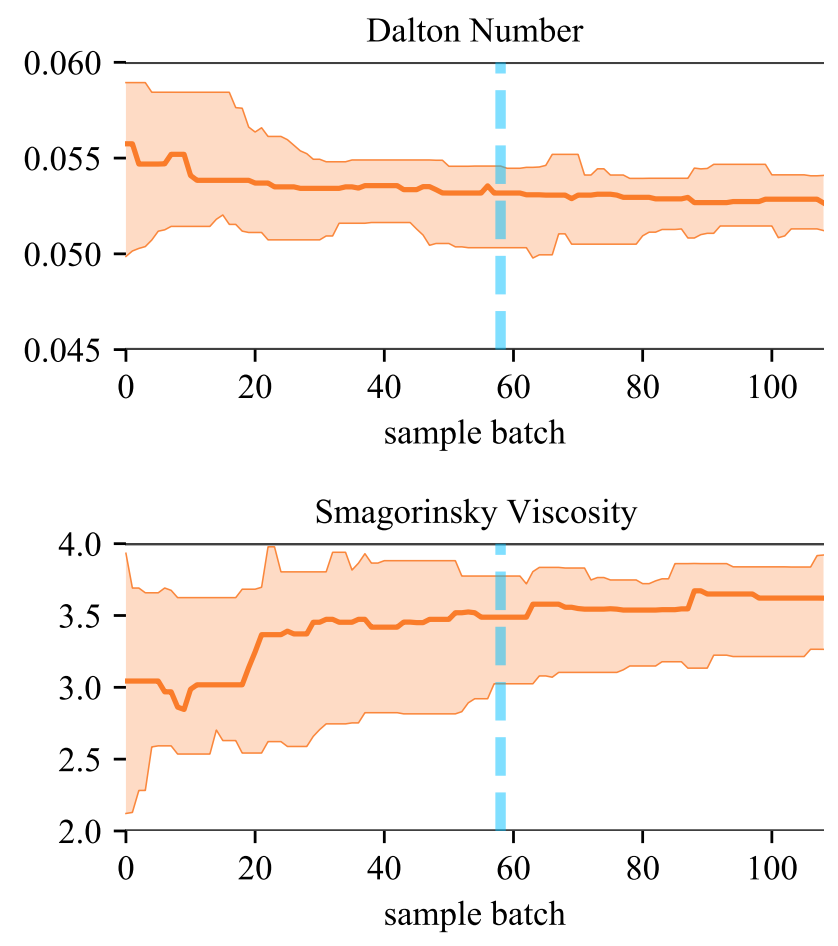

Figure 3. Evolution of the 16 Markov chain parameters. The solid lines indicate the median and the semi-transparent spreads indicate the 5\% - 95\% percentiles across multiple concurrent chains of the sampler. The vertical dashed blue line indicates the end of the specified burn-in period used to determine the posterior distribution.
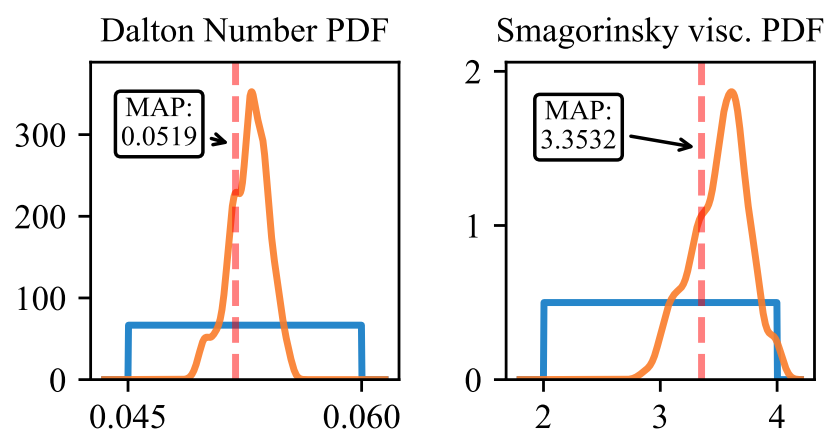

Figure 4. Marginal posterior (orange) and prior (blue) distributions of model parameters. The red dashed line indicates the maximum aposteriori parameter (MAP).

\subsection{In situ data assimilation results}

We present the results of the assimilated data predictions in this section, with the control run (CR) serving as the baseline for comparison. The data assimilation (DA) prediction was generated using the MAP values, given in Fig. 4 right. In addition, 


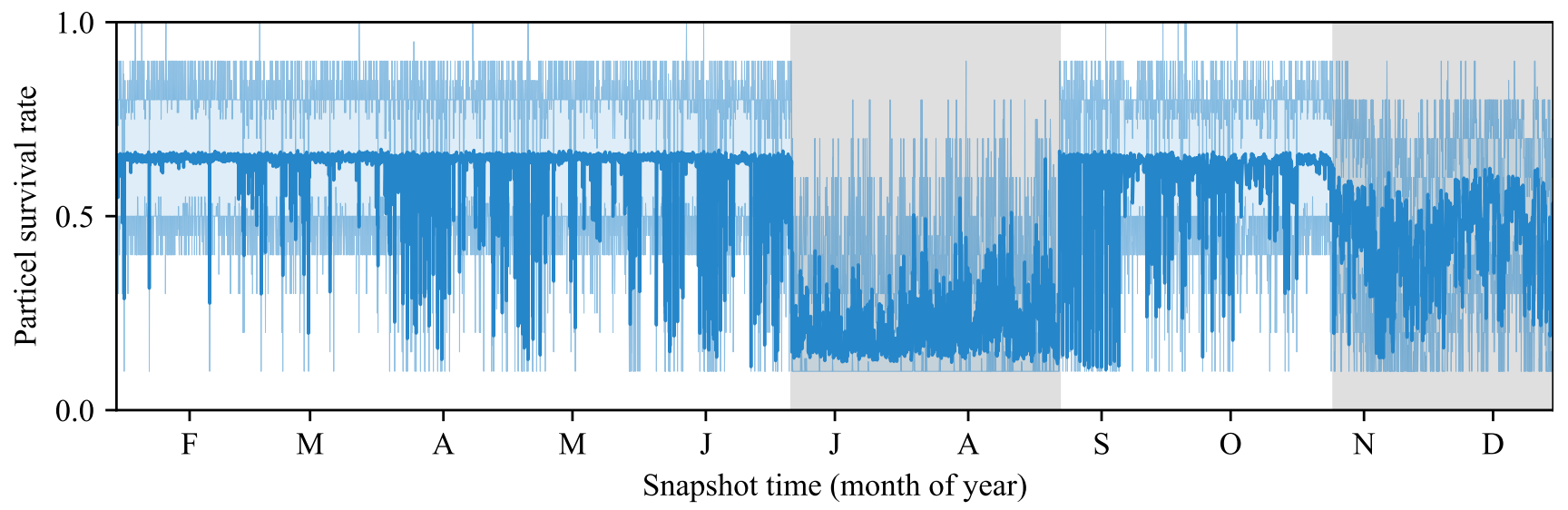

Figure 5. Particle survival rates (fraction of particles in the filter that are retained for continuing the DA) for each snapshot time in the PF likelihood estimator. The solid line indicates the mean, the semi-transparent lines indicate the $0 \%-100 \%$ percentiles. The periods of time when LéXPLORE data is available are shown as shaded gray regions.

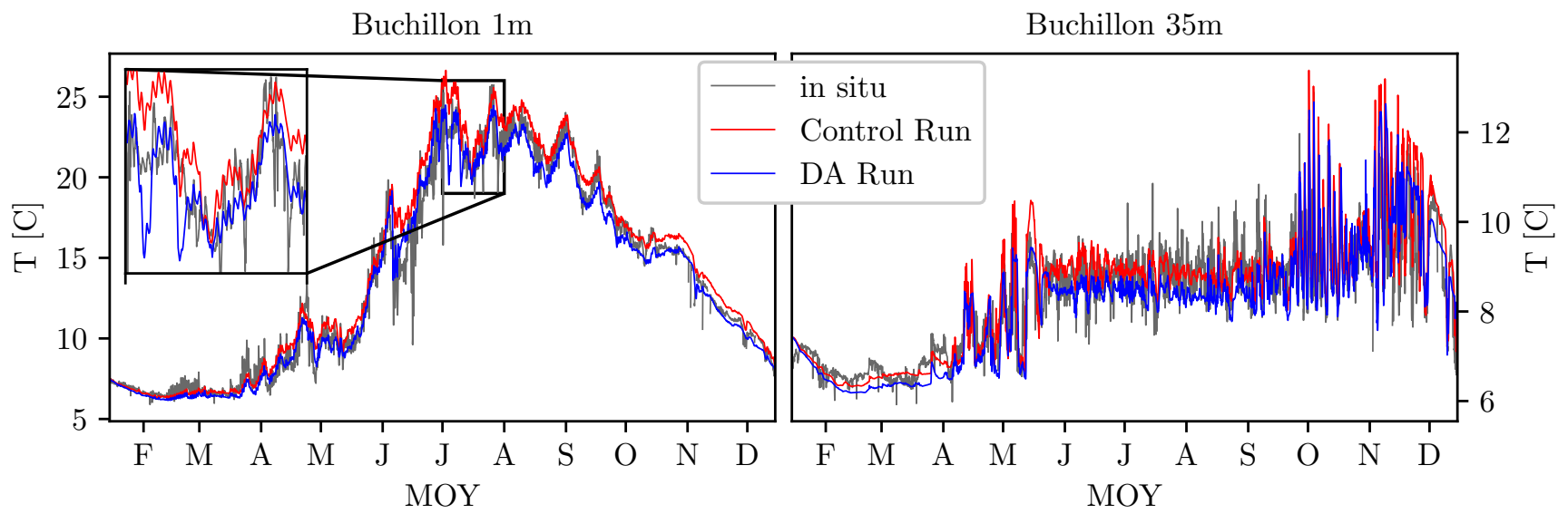

Figure 6. Evolution of temperature at the Buchillon station for the 1m (left) and 35m (right) sensors for the year 2019. The gray line is the in situ measurement, the red line corresponds to the control run, and the blue line is the data assimilated prediction. The small inset on the left shows the improvement from the assimilated run for the $1 \mathrm{~m}$ sensor in greater detail for July.

whenever possible, we compare the aggregate error metrics to the values given in Baracchini et al. (2020b) for their 2017 data (specifically we do not use their 2019 data, which exhibits a noticeable drift in the deeper layers of the lake in comparison to SHL2 observations). The performance of the model for the Buchillon, SHL2 and LéXPLORE in situ datasets are considered in this section.

Figure 6 shows the temperature for the Buchillon station at depths of $1 \mathrm{~m}$ (left) and 35m (right). The gray lines represent the measurement, the red line shows the CR, and the blue line is the calibrated and data assimilated (DA) run. As evident from the results at both depths, the $\mathrm{CR}$ already captures the seasonal variation and the high-frequency fluctuations quite accurately. The 
Table 3. Performance of the DA run across the different in situ datasets in comparison to the CR.

\begin{tabular}{|c||c|c||c|c|}
\hline Dataset & $\begin{array}{c}\text { CR } \\
\text { RMSE }\end{array}$ & $\begin{array}{c}\text { CR } \\
\text { MAE }\end{array}$ & $\begin{array}{c}\text { DA } \\
\text { RMSE }\end{array}$ & $\begin{array}{c}\text { DA } \\
\text { MAE }\end{array}$ \\
\hline \hline Buchillon $\left[{ }^{\circ} \mathrm{C}\right]$ & 0.95 & 0.61 & 0.77 & 0.54 \\
\hline LéXPLORE Temp $\left[{ }^{\circ} \mathrm{C}\right]$ & 1.26 & 0.81 & 1.01 & 0.67 \\
\hline LéXPLORE Vel $[\mathrm{m} / \mathrm{s}]$ & 0.033 & 0.023 & 0.029 & 0.020 \\
\hline SHL2 $\left[{ }^{\circ} \mathrm{C}\right]$ & 1.32 & 0.64 & 1.22 & 0.56 \\
\hline $\left.\mathrm{GE} 3{ }^{\circ} \mathrm{C}\right]$ & 1.45 & 0.84 & 1.25 & 0.76 \\
\hline
\end{tabular}
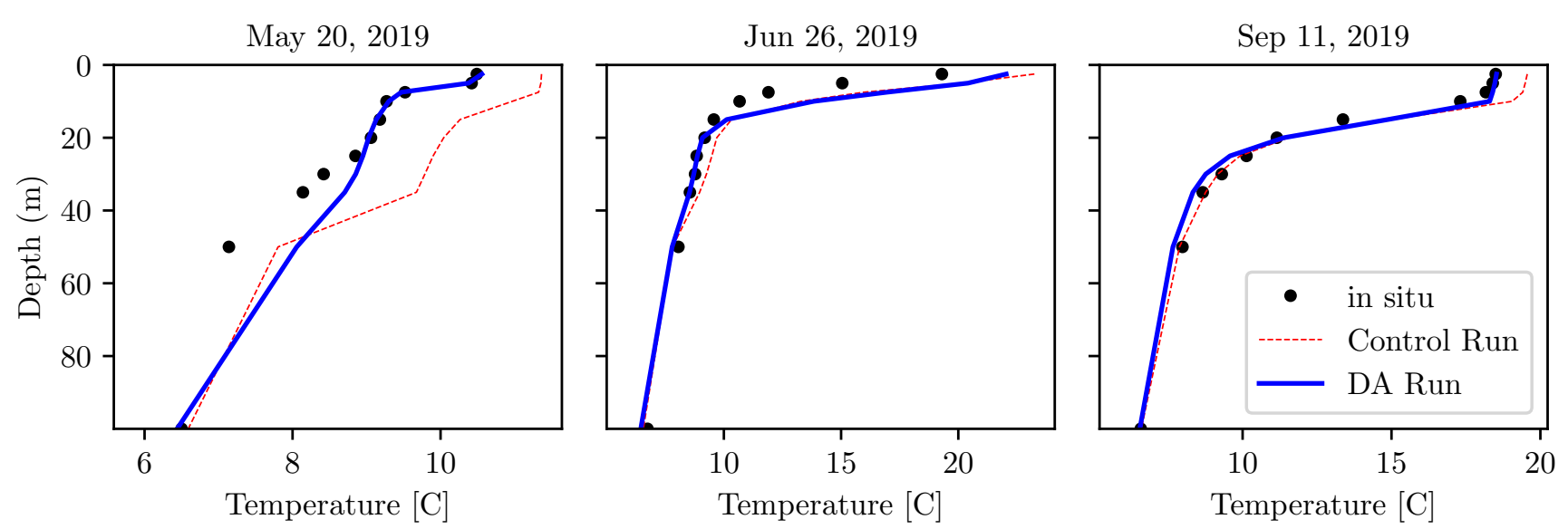

Figure 7. Vertical temperature profiles up to 100 meters deep at the SHL2 location. The black dots represent observations, the dashed red line is the control run, and the blue line shows the data assimilated run.

DA run improves model performance in the summer, where the CR under-predicts the near-surface temperature, as shown more clearly in the inset which focuses on the predictions for July. For the entire dataset, the RMSE decreases from $0.95{ }^{\circ} \mathrm{C}$ for the $\mathrm{CR}$ to $0.77^{\circ} \mathrm{C}$ for the DA run. Overall, the improvement in RMSE and mean average error (MAE) across the various datasets is $4-15 \%$, summarized in Table 3. The main source of performance improvement is the better resolution of the near-surface temperature in summer.

We now consider the vertical temperature column profiles from the SHL2 location, which provides monthly measurements at the deepest location in the lake. As both the CR and DA run predictions below 100m are in agreement with the measurements, we focus on the upper portion of the column. In Fig. 7, we analyze the performance of the models for a few selected snapshots. In the figure, the black dots represent measurements, the dashed red line is the $\mathrm{CR}$, and the thick blue line is the DA run. The results show that the DA run tracks the observed temperature near the surface with greater accuracy, which also results in better modeling of deeper-layer temperature. Below 60 meters the observations are followed quite accurately by both the CR and DA run, without any substantial difference between the models. 
Figure 8 compares the observed vertical column temperature at the LéXPLORE location to the DA run. The vast bulk of measurements for this location (both temperature and velocity) were obtained during two periods, as reflected in the figure: data for the period June 21 - August 11 is shown on the left, and the right side focuses on October 25 until December 15. For simplicity, we will refer to the respective time intervals as 'summer' and 'autumn'. Outside of those time periods, the measurements were sparse and therefore are omitted from comparison. The results show that the DA algorithm models the water column temperature quite accurately, and in particular, correctly reproduces the thermocline depth in the summer. Furthermore, the cooling cycle at the end of the year is captured quite well. Above the thermocline, the difference plots highlight that the DA run over-predicts the temperature in the summer months, which as a result generates a smaller and dissipating warm bias in autumn. The discrepancy could potentially be reconciled with a higher resolution model or more accurate Secchi depth estimates. In general, correcting temperature over-prediction in the subsurface turbulent layer is a difficult problem exhibited in many studies (Cimatoribus et al., 2018; Soulignac, Frédéric et al., 2018; Ye et al., 2020), without a clear consensus on the underlying causes for each case.

Due to the coarse horizontal resolution of the mesh, the data assimilation algorithm primarily focused on tracking temperature. As a result, the ADCP data played only a secondary role in determining the trajectory of the model. In Fig. 9, we present the kinetic energy spectra computed from the LéXPLORE ADCPs (gray lines) and the DA run (blue lines) based on different data sets. Figure 9 left, based on summer readings for the 15 meter sensor, shows excellent agreement for the kinetic energy variability above the semi-diurnal $(12 \mathrm{~h})$ mode. For the same sensor location, model predictions under-perform during the autumn (Fig. 9 right), indicating that the high frequency internal wave modes are not being resolved by the hydrodynamic model. A potentially large contributing factor for the discrepancies is the relatively coarse spatial resolution used in the model.

\subsection{LSWT assimilation using BiLSTM network}

On average, an LSWT image provided 209 usable pixels and significantly affected particle survival rates. For example, in Fig. 5 most of the low survival rates are due to the AVHRR data, especially noticeable in cases when LéXPLORE data is not available. In contrast to Baracchini et al. (2020b), where a highly selective criterion was applied, we use all the pixels which have an associated non-zero QF value. This allowed a much more frequent remote sensing data assimilation, with 798 (of 2092 total) images usable for the data assimilation period. The use of LSWT has enhanced the model predictions by only a $4 \%$ reduction of in situ RMSE. In Fig. 10, we show an example comparison between the observed LSWT (left), the hydrodynamic model bulk temperature (center), and the BiLSTM prediction (right) for a selected snapshot. The result shows that BiLSTM can predict the spatial variability and structure of LSWT images; although frequently it also generates an entirely different profile. In general, as these improvement results are not particularly informative, we instead focus on the overall performance of the assimilation model and the BiLSTM predictions.

The global training and performance of the BiLSTM model are summarized in Table 4. The training and testing used the means and spreads of the MeteoSwiss weather predictions in combination with Meteolakes mean bulk temperature. The results show that the network significantly improves predictions of LSWT for the training set, and achieves a 33\% reduction of RMSE for the test set. In the assimilated run, the bulk prediction difference is already small and only worse than BiLSTM 

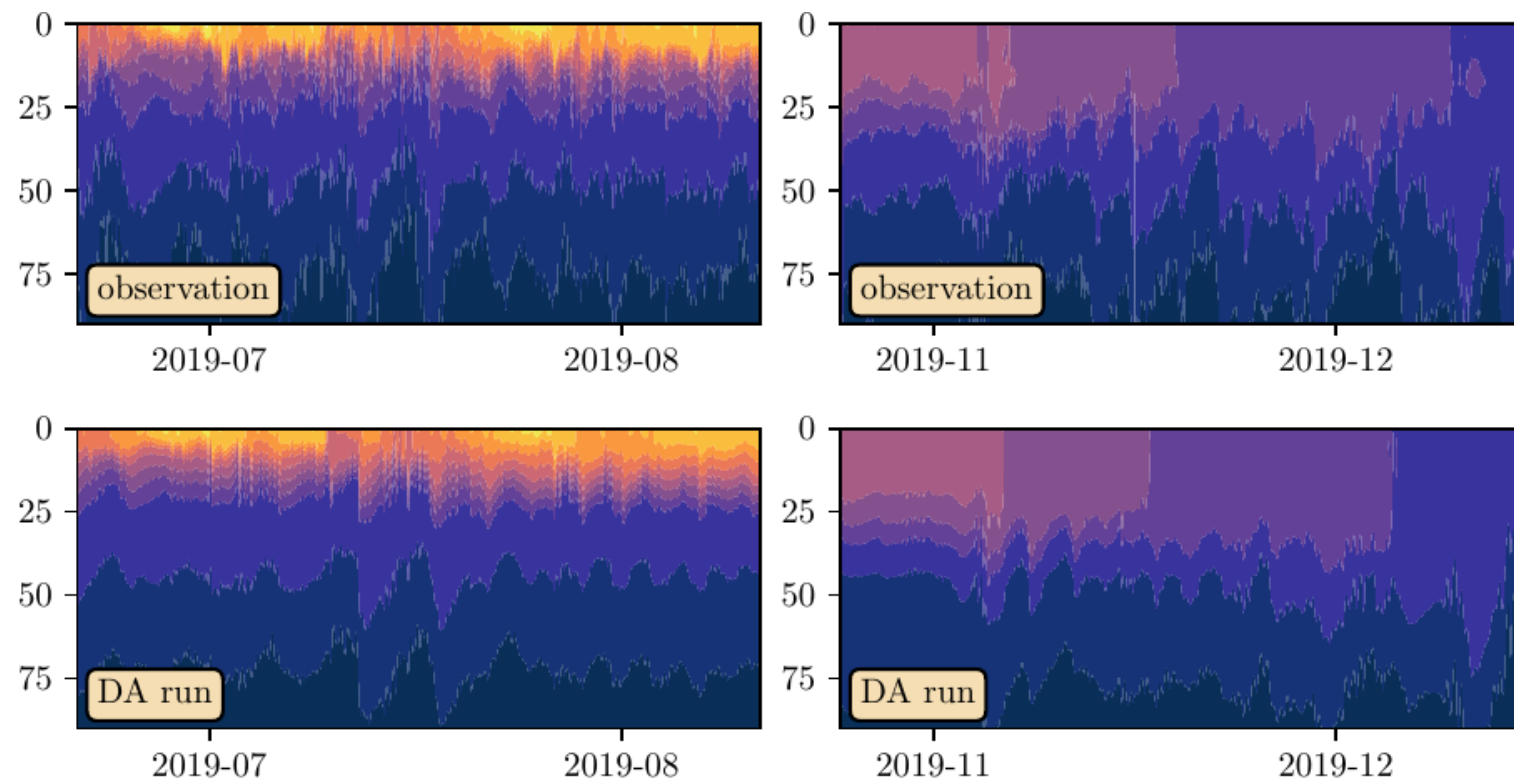

2019-07

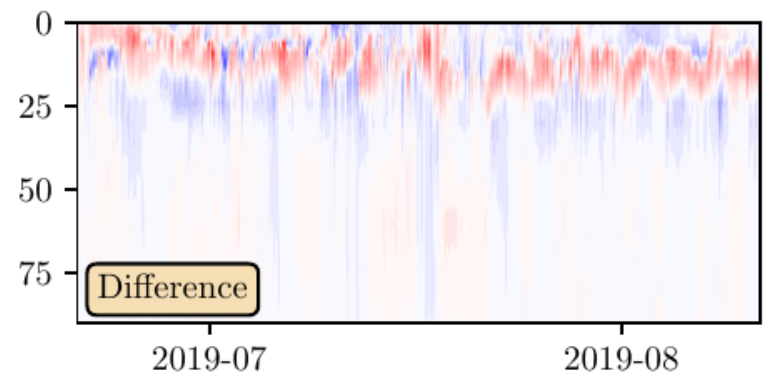

$2019-11$

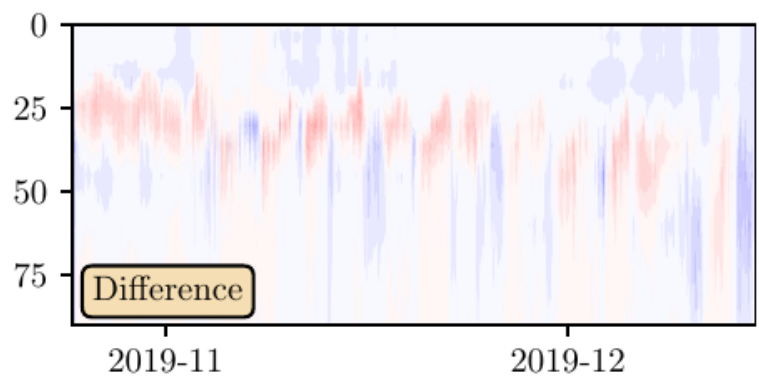

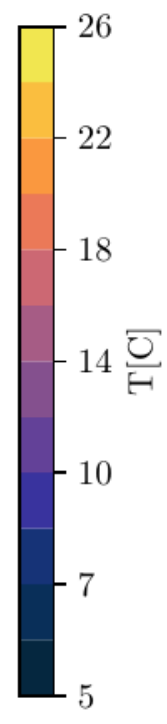

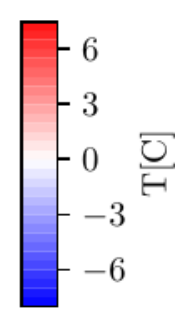

Figure 8. Vertical temperature profiles from the LéXPLORE sensors for summer months (left) and late autumn (right). The top row shows the dataset, the middle row shows the assimilated predictions. The bottom row shows the difference between the plots, with positive values indicating model over-prediction.

test set performance. In fact, the BiLSTM network increases the RMSE by about 10\%, which most likely is attributable to the differences between the training data and the assimilation process. In general, as LSWT measurements carry significant uncertainty (1.3-1.5 ${ }^{\circ} \mathrm{C}$ RMSE), the analytic capability is limited by the lack of exact skin temperature measurements for 2019.

Surprisingly, the predictive capability of the BiLSTM seems to improve for the LSWT pixels with an associated QF in the range 2-5 (42\% of the net pixel count), with an RMSE of $1.92{ }^{\circ} \mathrm{C}$ versus $2.11{ }^{\circ} \mathrm{C}$ for direct bulk comparison. For highest quality data (QF 6) however, BiLSTM does not improve performance. In Fig. 11 left, we analyze the performance of the BiLSTM (orange lines) against hydrodynamic model bulk predictions (in blue) for the different QFs by plotting mean RMSE with 10-90\% percentiles. The gray bar chart shows the total number of LSWT measurements for the particular QF level. Aside from QF 5, the bulk RMSE gradually increases for lower QFs, as expected. At the same time, the BiLSTM error is practically constant with lower uncertainty, indicating the network's capability to predict lower fidelity data. For QF 5, the discrepancy 

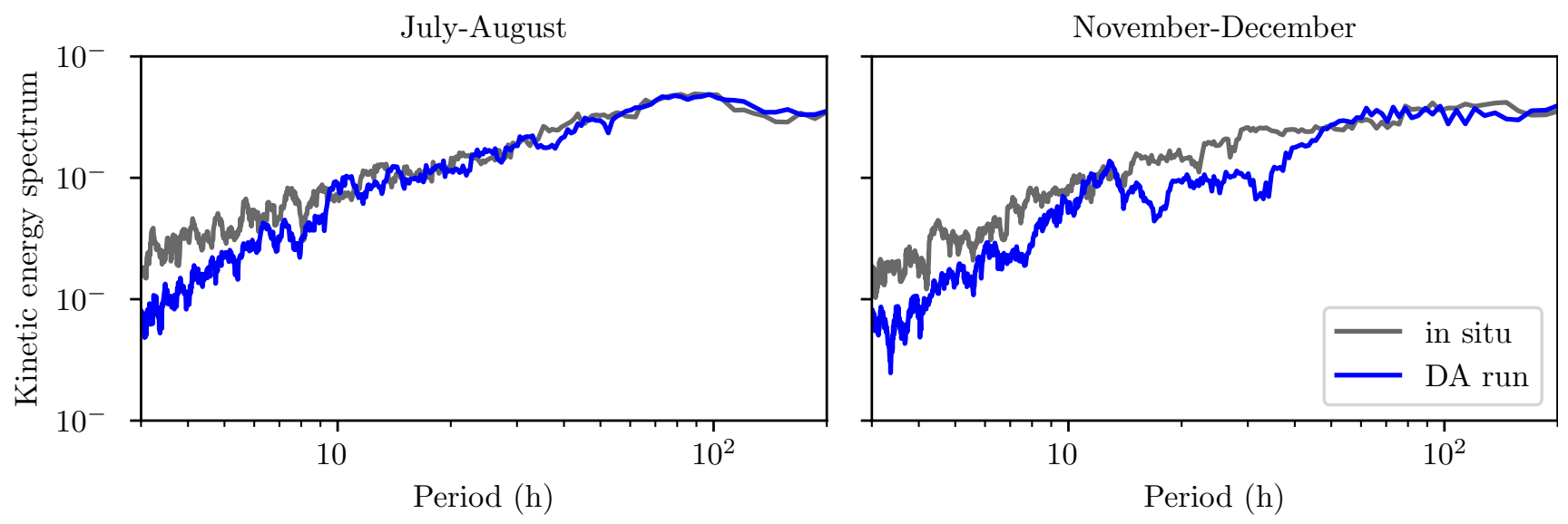

Figure 9. Kinetic energy spectra computed from the ADCP sensors at the LéXPLORE platform (gray) and from the DA run (blue). Profiles based on 15 meter deep dynamics use July-August data for the left figure and November-December data for the right figure.

\section{LSWT snapshot for Aug 8, 16:00}

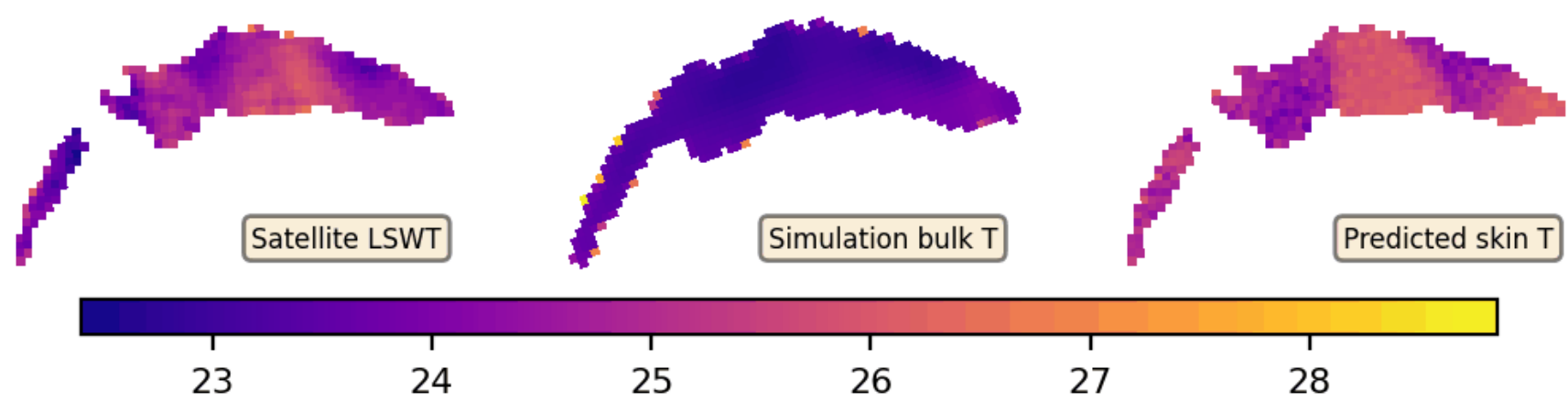

Figure 10. A comparison of LSWT snapshot from August 8, 2019 to the DA bulk and BiLSTM skin temperature predictions.

Table 4. Results of the BiLSTM model training (left two columns, with bulk temperature from Meteolakes (Baracchini et al., 2020b)), and performance in DA Run (right column).

\begin{tabular}{|c||c|c||c|}
\hline & $\begin{array}{c}\text { Training set } \\
\text { (w. Meteolakes } \\
\text { data) }\end{array}$ & $\begin{array}{c}\text { Test set } \\
\text { (w. Meteolakes } \\
\text { data) }\end{array}$ & $\begin{array}{c}\text { DA Run } \\
\text { (SPUX- } \\
\text { MITgcm) }\end{array}$ \\
\hline \hline $\begin{array}{c}\text { Bulk } \\
\text { RMSE }\end{array}$ & 3.00 & 2.37 & 1.85 \\
\hline $\begin{array}{c}\text { BiLSTM } \\
\text { RMSE }\end{array}$ & 1.33 & 1.60 & 1.99 \\
\hline
\end{tabular}




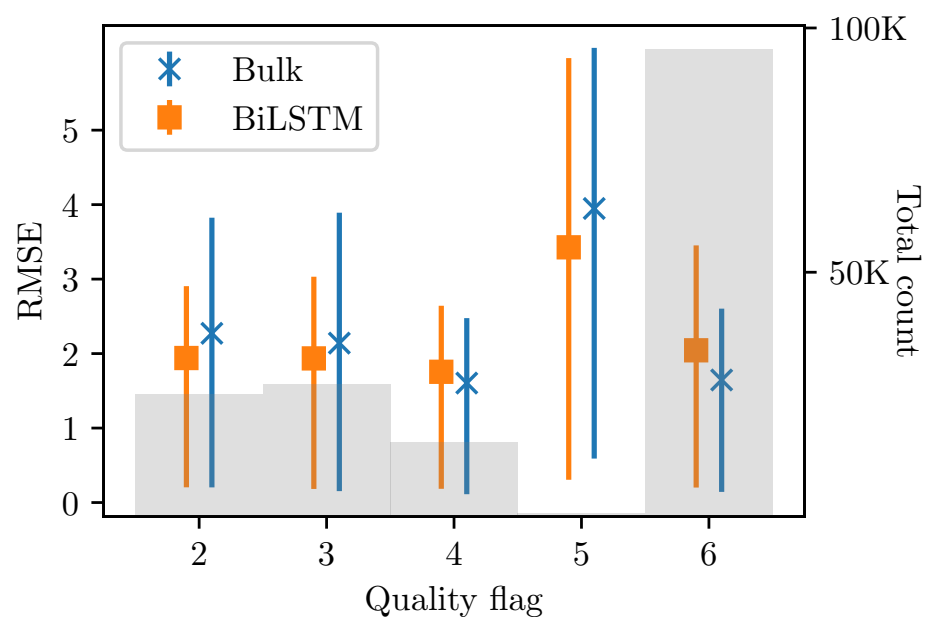

Spatial distribution of QF 5

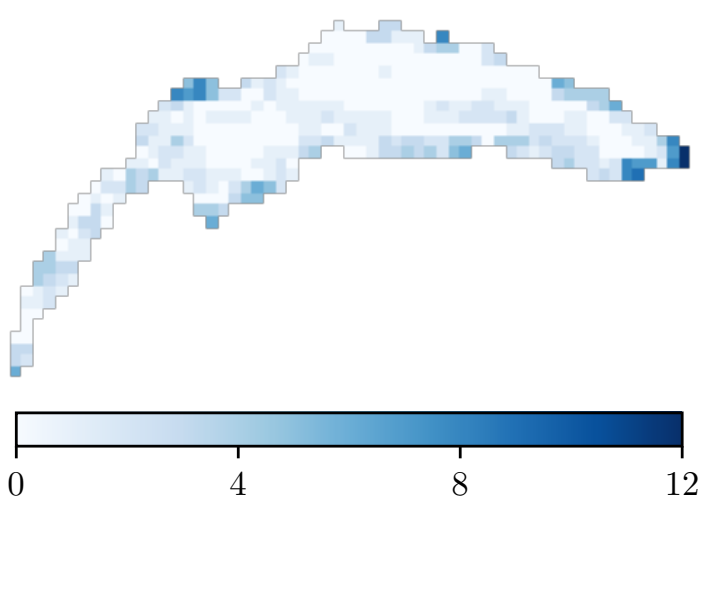

Figure 11. Analysis of Bi-LSTM performance in the DA run with respect to the different quality flags. Left: LSWT difference with respect to bulk temperature (in blue) and BiLSTM predictions (in orange) as a function of QF. The faint gray bars show the total number of LSWT measurements associated with the QF. Right: spatial distribution of LSWT measurement availability for QF 5.

nearly doubles, indicating a significant issue with this LSWT data subset. In contrast to the relatively uniform spatial frequency of other QFs on the lake, Fig. 11 right shows that most of the associated measurements occurred near the shore, which are harder to predict accurately due to the resolution of the hydrodynamic model. However, as LSWT pixels with associated QF 5 are extremely rare (Fig. 11 left), they do not significantly contribute to the overall result.

To enable uncertainty quantification (UQ) for the PF, each individual bulk-to-skin prediction is also equipped with a normal uncertainty distribution, as described in Sect. 2.4.3. Since the network used hour of the day and weather prediction uncertainty as part of its training, we can expect some spatio-temporal variation in the predicted spreads. Therefore, we analyze those two factors here. In Fig. 12 left, we show the hourly mean BiLSTM prediction difference with LSTM data as solid blue line, and the 10\%-90\% percentiles of the BiLSTM UQ as shaded blue regions. The results suggest that neural network UQ is capable of slightly better predicting the spreads for the different times of the day. In contrast, the default LSWT error model provides relatively uniform uncertainties across all times of the day, regardless of MAE (Fig. 12 right). In general, the improvement is however quite mild, potentially due to the low spatial resolution of the model.

Finally, we consider the spatial pattern of BiLSTM predictions, and in particular, examine whether its UQ can predict regions of the lake with larger model discrepancies. In Fig. 13 left, we present mean BiLSTM prediction RMSE for the different pixel locations over the lake. As can be expected, the best performance is obtained for off-shore pixels in the eastern portion of the lake (Grand Lac). The predictive capability of the network reduces nearshore, and especially in the western portion (in the Petit Lac). The BiLSTM uncertainty predictions follow a much similar pattern (Fig. 13 right), including the large increases in uncertainty near the north shores. In part, this increase can most likely be attributed to the greater uncertainty in the atmospheric 

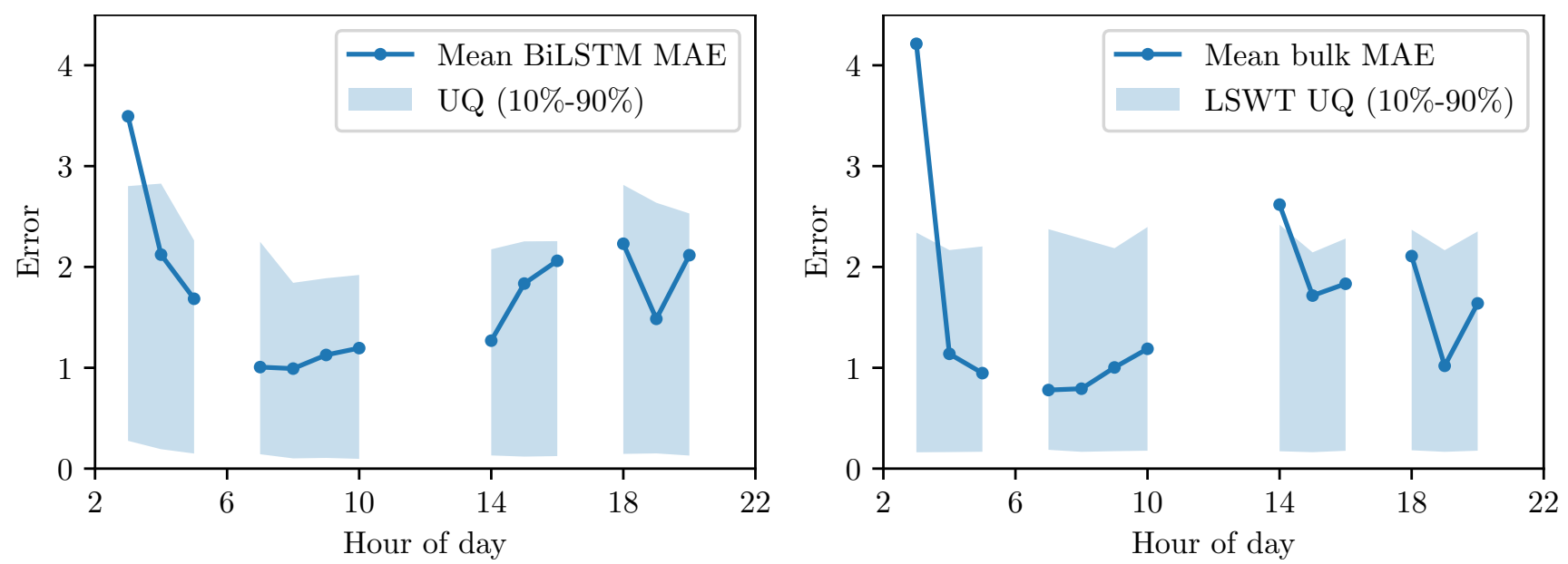

Figure 12. Left: mean BiLSTM prediction error (dark blue line) and the 10\%-90\% percentiles of the uncertainties predicted by Bi-LSTM (shaded areas) for the different hours of the day. Right: mean bulk temperature prediction error (dark blue line) and the 10\%-90\% uncertainty estimates based on Lieberherr and Wunderle (2018).
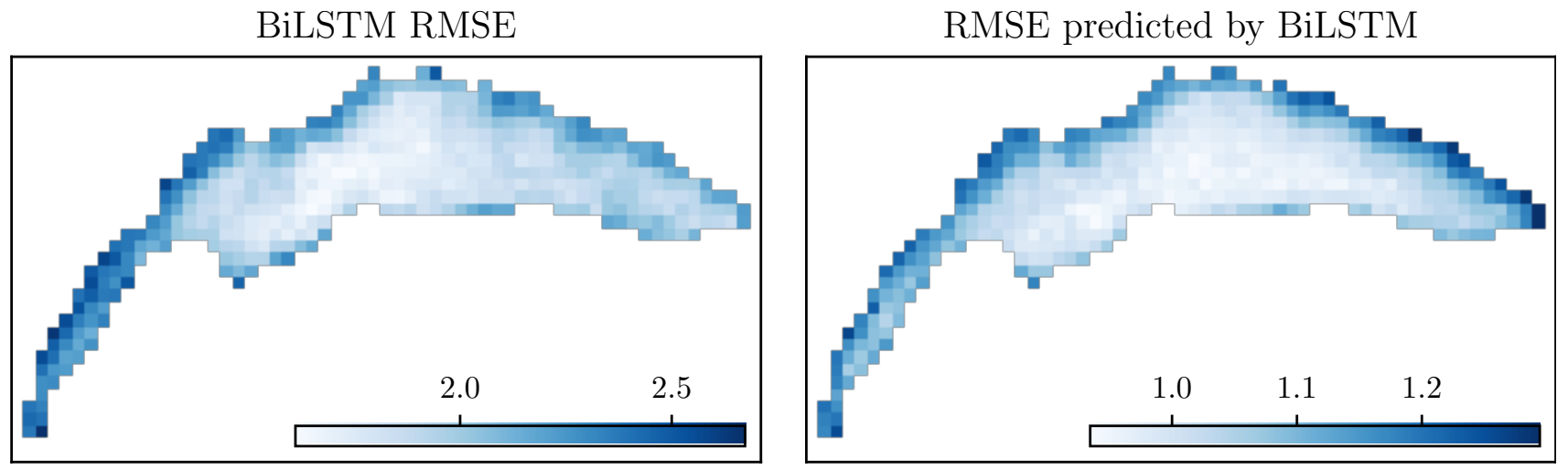

Figure 13. Spatial distribution for the BiLSTM RMSE (left) and the RMSE predicted by the BiLSTM.

weather conditions near the land-water interface, as well as the fact that the majority of observations for training BiLSTM come from off-shore in the Grand Lac, which would likely create a bias in the model predictions.

\section{Conclusions}

We presented the SPUX-MITgcm framework, a novel approach to the calibration of hydrodynamic model for highly spatiotemporally heterogeneous observational dataset. The inference makes use of the ensemble affine invariant sampler (EMCEE) to infer the distribution of model parameters coupled with a particle filter (PF) for stochasticity in atmospheric forcing. The PF relied on resampling existing trajectories based on their observational likelihoods to infer the most probable weather conditions 
https://doi.org/10.5194/gmd-2021-305

Preprint. Discussion started: 12 November 2021

(c) Author(s) 2021. CC BY 4.0 License.

(c) (i)

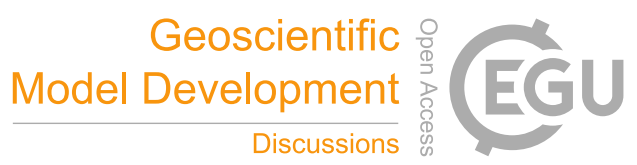

over the lake. As a result, the PF generated physically realistic trajectories (at least, with respect to the hydrodynamic model). In addition, to enable the proper assimilation of remotely sensed lake surface temperature, we developed a Bi-directional Long Short-Term Memory network for estimating lake skin temperature based on a history of weather and bulk temperature predictions.

The particle filter provides a relatively small improvement to model predictions (in contrast to other popular data assimilation schemes), but at no cost to the quality of the physical model. However, this approach requires a highly robust hydrodynamic model, as its corrective powers are limited. Despite the improvements, this approach is quite computationally costly, especially as a tool for inferring model parameters. In addition, as discussed in the supplementary material, the sampler has significant difficulty with calibrating certain model parameters (although this issue could potentially be mitigated with a better error model). Therefore, we feel that a computationally cheaper method for parameter estimation (for example, an optimization algorithm instead of a sampler) might be the more productive approach. At the same time, an improved version of the particle filtering approach could provide a powerful option for operational forecasting models.

Code and data availability. The code used for these simulations, and an example portion of the data, are openly available. The SPUX source code is available at https://doi.org/10.5281/zenodo.5638313, the modified MITgcm repository can be found at https://doi.org/10. 5281/zenodo.5634042, and the repository for handling MI Tgcm runs is at https://doi.org/10.5281/zenodo.5637216. While we cannot openly publish the forcing data, a representative snapshot is available at https://renkulab.io/gitlab/artur.safin/datalakes-observational-data-snapshot, and the 3D mean temperature and velocity predictions can be accessed at https://doi.org/10.5281/zenodo.5642898. Finally, the reproducibility of the computational environment is enabled through RENKU (Swiss Data Science Center, 2021), an online platform for the storage, tracking and replication of numerical codes, which enables users to launch the container directly from the RENKU site. The entire SPUX-MITgcm repository and the computational environment is available as a docker at https://renkulab.io/projects/artur.safin/DatalakesHydrodynamics, and an example of running the inference is in the supplementary material.

Author contributions. JS and DB designed the research, AS implemented the data assimilation framework and ran the inference. Šukys extended SPUX package to be compatible with MITgcm. FO implemented and trained the BiLSTM network. DB and CLR advised with the hydrodynamic model. All authors assisted with discussions, dataset management and manuscript preparation.

Competing interests. The authors declare that they have no conflict of interest.

Acknowledgements. This work was funded by the Swiss Data Science Center (SDSC Project DATALAKES C17-17) and Eawag Discretionary Funding. We would like to thank the entire team from LéXPLORE platform, for their administrative and technical support and for LéXPLORE core dataset. We also acknowledge LéXPLORE five partner institutions: Eawag, EPFL, University of Geneva, University of 
https://doi.org/10.5194/gmd-2021-305

Preprint. Discussion started: 12 November 2021

(C) Author(s) 2021. CC BY 4.0 License.

(c) (1)

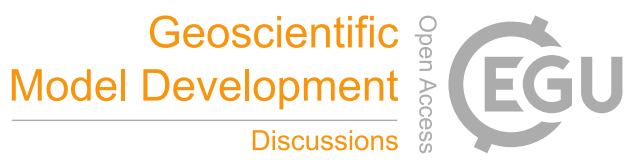

Lausanne and CARRTEL (INRAE-USMB). For SHL2 data, we acknowledge the Observatory of alpine LAkes (OLA), SOERE OLA-IS, AnaEE-France, INRA of Thonon-les-Bains and CIPEL. We would like to thank the Swiss Federal Office for the Environment (BAFU) for river and water level data and Stefan Wunderle and the Remote Sensing group at the University of Bern for LSWT data. The authors would also like to thank Marco Bacci, Theo Baracchini, Fernando Perez-Cruz, Eric Bouillet and Daniel Odermatt for helpful discussions. 


\section{References}

Adcroft, A., Hill, C., and Marshall, J.: Representation of Topography by Shaved Cells in a Height Coordinate Ocean Model, Monthly Weather Review, 125, 2293 - 2315, https://doi.org/10.1175/1520-0493(1997)125<2293:ROTBSC>2.0.CO;2, 1997.

Alappattu, D. P., Wang, Q., Yamaguchi, R., Lind, R. J., Reynolds, M., and Christman, A. J.: Warm layer and cool skin corrections for bulk water temperature measurements for air-sea interaction studies, Journal of Geophysical Research: Oceans, 122, 6470-6481, https://doi.org/https://doi.org/10.1002/2017JC012688, 2017.

Anderson, E. J., Fujisaki-Manome, A., Kessler, J., Lang, G. A., Chu, P. Y., Kelley, J. G., Chen, Y., and Wang, J.: Ice Forecasting in the Next-Generation Great Lakes Operational Forecast System (GLOFS), Journal of Marine Science and Engineering, 6, https://doi.org/10.3390/jmse6040123, 2018.

Andrieu, C., Doucet, A., and Holenstein, R.: Particle Markov chain Monte Carlo methods, Journal of the Royal Statistical Society: Series B (Statistical Methodology), 72, 269-342, https://doi.org/https://doi.org/10.1111/j.1467-9868.2009.00736.x, 2010.

Baracchini, T.: From observations to 3D forecasts: Data assimilation for high resolution lakes monitoring, Ph.D. thesis, EPFL, Lausanne, https://doi.org/10.5075/epfl-thesis-9475, 2019.

Baracchini, T., Chu, P. Y., Šukys, J., Lieberherr, G., Wunderle, S., Wüest, A., and Bouffard, D.: Data assimilation of in situ and satellite remote sensing data to 3D hydrodynamic lake models: a case study using Delft3D-FLOW v4.03 and OpenDA v2.4, Geoscientific Model Development, 13, 1267-1284, https://doi.org/10.5194/gmd-13-1267-2020, 2020a.

Baracchini, T., Wüest, A., and Bouffard, D.: Meteolakes: An operational online three-dimensional forecasting platform for lake hydrodynamics, Water Research, 172, 115 529, https://doi.org/https://doi.org/10.1016/j.watres.2020.115529, 2020 b.

Bouffard, D. and Lemmin, U.: Kelvin waves in Lake Geneva, Journal of Great Lakes Research, 39 , 637 - 645, https://doi.org/https://doi.org/10.1016/j.jglr.2013.09.005, 2013.

Bouffard, D. and Wüest, A.: Convection in Lakes, Annual Review of Fluid Mechanics, 51, 189-215, https://doi.org/10.1146/annurev-fluid010518-040506, 2019.

Bouffard, D., Kiefer, I., Wüest, A., Wunderle, S., and Odermatt, D.: Are surface temperature and chlorophyll in a large deep lake related? An analysis based on satellite observations in synergy with hydrodynamic modelling and in-situ data, Remote Sensing of Environment, 209 , 510-523, https://doi.org/https://doi.org/10.1016/j.rse.2018.02.056, 2018.

Chen, C., Beardsley, R. C., and Cowles, G.: An Unstructured Grid, Finite-Volume Coastal Ocean Model (FVCOM) System, Oceanography, 19, 78-89, https://doi.org/10.5670/oceanog.2006.92, 2006.

Chu, P. Y., Kelley, J. G. W., Mott, G. V., Zhang, A., and Lang, G. A.: Development, implementation, and skill assessment of the NOAA/NOS Great Lakes Operational Forecast System, Ocean Dynamics, 61, 1305-1316, https://doi.org/10.1007/s10236-011-0424-5, 2011.

Cimatoribus, A. A., Lemmin, U., Bouffard, D., and Barry, D. A.: Nonlinear Dynamics of the Nearshore Boundary Layer of a Large Lake (Lake Geneva), Journal of Geophysical Research: Oceans, 123, 1016-1031, https://doi.org/10.1002/2017JC013531, 2018.

Cogley, J. G.: The Albedo of Water as a Function of Latitude, Monthly Weather Review, 107, 775-781, https://doi.org/10.1175/15200493(1979)107<0775:TAOWAA>2.0.CO;2, 1979.

Deltares: Delft3D-FLOW user manual, 2013.

Fink, G., Schmid, M., and Wüest, A.: Large lakes as sources and sinks of anthropogenic heat: Capacities and limits, Water Resources Research, 50, 7285-7301, https://doi.org/10.1002/2014WR015509, 2014. 
https://doi.org/10.5194/gmd-2021-305

Preprint. Discussion started: 12 November 2021

(c) Author(s) 2021. CC BY 4.0 License.

(c) (i)

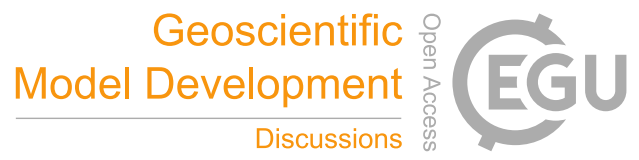

Gaudard, A., Råman Vinnå, L., Bärenbold, F., Schmid, M., and Bouffard, D.: Toward an open access to high-frequency lake modeling and statistics data for scientists and practitioners - the case of Swiss lakes using Simstrat v2.1, Geoscientific Model Development, 12, 3955-3974, https://doi.org/10.5194/gmd-12-3955-2019, 2019.

Goodman, J. and Weare, J.: Ensemble samplers with affine invariance, Communications in Applied Mathematics and Computational Science, 5, 65-80, https://doi.org/10.2140/camcos.2010.5.65, 2010.

Griffies, S. M. and Hallberg, R. W.: Biharmonic Friction with a Smagorinsky-Like Viscosity for Use in Large-Scale Eddy-Permitting Ocean Models, Monthly Weather Review, 128, 2935 - 2946, https://doi.org/10.1175/1520-0493(2000)128<2935:BFWASL>2.0.CO;2, 2000.

Gustafsson, N., Janjić, T., Schraff, C., Leuenberger, D., Weissmann, M., Reich, H., Brousseau, P., Montmerle, T., Wattrelot, E., Bučánek, A., Mile, M., Hamdi, R., Lindskog, M., Barkmeijer, J., Dahlbom, M., Macpherson, B., Ballard, S., Inverarity, G., Carley, J., Alexander, C., Dowell, D., Liu, S., Ikuta, Y., and Fujita, T.: Survey of data assimilation methods for convective-scale numerical weather prediction at operational centres, Quarterly Journal of the Royal Meteorological Society, 144, 1218-1256, https://doi.org/https://doi.org/10.1002/qj.3179, 2018.

Kendall, A. and Gal, Y.: What Uncertainties Do We Need in Bayesian Deep Learning for Computer Vision?, in: Advances in Neural Information Processing Systems, edited by Guyon, I., Luxburg, U. V., Bengio, S., Wallach, H., Fergus, R., Vishwanathan, S., and Garnett, R., vol. 30, Curran Associates, Inc., https://proceedings.neurips.cc/paper/2017/file/2650d6089a6d640c5e85b2b88265dc2b-Paper.pdf, 2017.

Kilpatrick, K. A., Podestá, G. P., and Evans, R.: Overview of the NOAA/NASA advanced very high resolution radiometer Pathfinder algorithm for sea surface temperature and associated matchup database, Journal of Geophysical Research: Oceans, 106, 9179-9197, https://doi.org/https://doi.org/10.1029/1999JC000065, 2001.

Lahoz, W. A. and Schneider, P.: Data assimilation: making sense of Earth Observation, Frontiers in Environmental Science, 2, 16, https://doi.org/10.3389/fenvs.2014.00016, 2014

Large, W. G., McWilliams, J. C., and Doney, S. C.: Oceanic vertical mixing: A review and a model with a nonlocal boundary layer parameterization, Reviews of Geophysics, 32, 363-403, https://doi.org/10.1029/94RG01872, 1994.

Lieberherr, G. and Wunderle, S.: Lake Surface Water Temperature Derived from 35 Years of AVHRR Sensor Data for European Lakes, Remote Sensing, 10, https://doi.org/10.3390/rs10070990, 2018.

415 McDougall, T. J., Jackett, D. R., Wright, D. G., and Feistel, R.: Accurate and Computationally Efficient Algorithms for Potential Temperature and Density of Seawater, Journal of Atmospheric and Oceanic Technology, 20, 730-741, https://doi.org/10.1175/15200426(2003)20<730:AACEAF>2.0.CO;2, 2003.

Prather, M. J.: Numerical advection by conservation of second-order moments, Journal of Geophysical Research: Atmospheres, 91, 66716681, https://doi.org/10.1029/JD091iD06p06671, 1986.

Rahaghi, A. I., Lemmin, U., Cimatoribus, A., Bouffard, D., Riffler, M., Wunderle, S., and Barry, D. A.: Improving surface heat flux estimation for a large lake through model optimization and two-point calibration: The case of Lake Geneva, Limnology and Oceanography: Methods, 16, 576-593, https://doi.org/10.1002/lom3.10267, 2018.

Riffler, M., Lieberherr, G., and Wunderle, S.: Lake surface water temperatures of European Alpine lakes (1989-2013) based on the Advanced Very High Resolution Radiometer (AVHRR) 1 km data set, Earth System Science Data, 7, 1-17, https://doi.org/10.5194/essd-7-1-2015, 2015.

Soulignac, F., Anneville, O., Bouffard, D., Chanudet, V., Dambrine, E., Guénand, Y., Harmel, T., Ibelings, B. W., Trevisan, D., Uittenbogaard, R., and Danis, P.: Contribution of 3D coupled hydrodynamic-ecological modeling to assess the representativeness of a sampling protocol for lake water quality assessment, Knowl. Manag. Aquat. Ecosyst., p. 42, https://doi.org/10.1051/kmae/2019034, 2019. 
https://doi.org/10.5194/gmd-2021-305

Preprint. Discussion started: 12 November 2021

(c) Author(s) 2021. CC BY 4.0 License.

(c) (1)

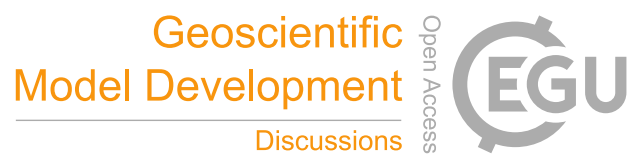

Soulignac, Frédéric, Danis, P.-A., Bouffard, D., Chanudet, V., Dambrine, E., Guénand, Yann, Harmel, T., Ibelings, B. W., Trevisan, D., Uittenbogaard, R., and Anneville, O.: Using 3D modeling and remote sensing capabilities for a better understanding of spatio-temporal heterogeneities of phytoplankton abundance in large lakes, Journal of Great Lakes Research, 44, 756 - 764, https://doi.org/https://doi.org/10.1016/j.jglr.2018.05.008, 2018.

Swiss Data Science Center: RENKU, https://renkulab.io/, 2021.

van Leeuwen, P. J., Künsch, H. R., Nerger, L., Potthast, R., and Reich, S.: Particle filters for high-dimensional geoscience applications: A review, Quarterly Journal of the Royal Meteorological Society, 145, 2335-2365, https://doi.org/https://doi.org/10.1002/qj.3551, 2019.

Verburg, P. and Antenucci, J. P.: Persistent unstable atmospheric boundary layer enhances sensible and latent heat loss in a tropical great lake: Lake Tanganyika, Journal of Geophysical Research: Atmospheres, 115, https://doi.org/10.1029/2009JD012839, 2010.

Vinnå, L. R., Medhaug, I., Schmid, M., and Bouffard, D.: The vulnerability of lakes to climate change along an altitudinal gradient, Communications Earth \& Environment, 2, https://doi.org/10.1038/s43247-021-00106-w, 2021.

440 Wick, G. A., Emery, W. J., Kantha, L. H., and Schlüssel, P.: The Behavior of the Bulk - Skin Sea Surface Temperature Difference under Varying Wind Speed and Heat Flux, Journal of Physical Oceanography, 26, 1969 - 1988, https://doi.org/10.1175/15200485(1996)026<1969:TBOTBS>2.0.CO;2, 1996.

Wüest, A. and Lorke, A.: SMALL-SCALE HYDRODYNAMICS IN LAKES, Annual Review of Fluid Mechanics, 35, 373-412, https://doi.org/10.1146/annurev.fluid.35.101101.161220, 2003.

445 Wüest, A., Bouffard, D., Guillard, J., Ibelings, B. W., Lavanchy, S., Perga, M.-E., and Pasche, N.: LéXPLORE: A floating laboratory on Lake Geneva offering unique lake research opportunities, WIREs Water, 8, e1544, https://doi.org/https://doi.org/10.1002/wat2.1544, 2021.

Ye, X., Chu, P. Y., Anderson, E. J., Huang, C., Lang, G. A., and Xue, P.: Improved thermal structure simulation and optimized sampling strategy for Lake Erie using a data assimilative model, Journal of Great Lakes Research, 46, 144-158, https://doi.org/https://doi.org/10.1016/j.jglr.2019.10.018, 2020.

Šukys, J. and Bacci, M.: SPUX Framework: a Scalable Package for Bayesian Uncertainty Quantification and Propagation, https://arxiv.org/ abs/2105.05969, 2021. 